\title{
Correction to: Development of dual-emission cluster of Ag atoms for genetically modified organisms detection
}

\author{
Mohsen Alipour ${ }^{1}$ - Shirin Jalili ${ }^{2} \cdot$ Hadi Shirzad ${ }^{2}$ Ehsan Ansari Dezfouli ${ }^{3} \cdot$ Mohamad Hassan Fouani $^{3}$. \\ Amir Amiri Sadeghan ${ }^{4}$. Hassan Bardania ${ }^{5,6}$. Saman Hosseinkhani ${ }^{3,7}$
}

Published online: 17 November 2020

(C) Springer-Verlag GmbH Austria, part of Springer Nature 2020

\section{Correction to: Microchimica Acta \\ https://doi.org/10.1007/s00604-020-04591-2}

The published version of this article, unfortunately, contains error in the affiliation. The authors express their sincere apology and corrected the affiliations in this article.

Moreover, the following statement should be added to Acknowledgments:

"This work was financially supported by Jahrom University of Medical Sciences. This study was also approved by the Ethics Committee of Jahrom University of Medical Sciences (Approval ID: IR.JUMS.REC.1397.071)."

The original article has been corrected.

Publisher's note Springer Nature remains neutral with regard to jurisdictional claims in published maps and institutional affiliations.

The online version of the original article can be found at https://doi.org/ $10.1007 / \mathrm{s} 00604-020-04591-2$

Mohsen Alipour

m.alipour@jums.ac.ir

$\triangle$ Saman Hosseinkhani

saman_h@modares.ac.ir

1 Department of Advanced Medical Sciences \& Technologies, School of Medicine, Jahrom University of Medical Sciences, Jahrom, Iran

2 Research Institute of Police Science \& Social Studies, Tehran, Iran

3 Department of Nanobiotechnology, Faculty of Biological Sciences, Tarbiat Modares University, Tehran, Iran

4 Tuberculosis and Lung Diseases Research Center, Tabriz University of Medical Sciences, Tabriz, Iran

5 Cellular and Molecular Research Center, Yasuj University of Medical Sciences, Yasuj, Iran

6 Clinical Research Development Unit, Imamsajad Hospital, Yasuj University of Medical Sciences, Yasuj, Iran

7 Department of Biochemistry, Faculty of Biological Sciences, Tarbiat Modares University, Tehran, Iran 